\title{
EFFICIENT CALCULATION OF ELECTRON STATES IN SELF-ASSEMBLED QUANTUM DOTS: APPLICATION TO AUGER RELAXATION
}

\author{
D. Chaney, M. Roy and P. A. Maksym \\ University of Leicester \\ Leicester LE1 7RH, UK
}

\begin{abstract}
An efficient method for calculation of self-assembled dot states within the effective mass approximation is described and its application to the calculation of Auger relaxation rates is detailed. The method is based on expansion of the dot states in a harmonic oscillator basis whose parameters are optimised to improve the convergence rate. This results in at least an order of magnitude reduction in the number of basis states required to represent electron states accurately compared to the conventional plane wave approach. Auger relaxation rates are calculated for harmonic oscillator model states and exact states for various pyramidal models. The dipole approximation, previously used to calculate Auger rates, is found to be inaccurate by a factor of around 2-3. The harmonic oscillator states do not reproduce the rates for the more realistic pyramidal models very well and even within the set of pyramidal models variations in the dot shape and size can change the rates by up to an order of magnitude. Typical Auger relaxation rates are on a picosecond timescale but the actual value is strongly dependent on the density of electrons outside the dot.
\end{abstract}

Keywords: Self-assembled dot, electron states, Auger relaxation

\section{Introduction}

Self-assembled quantum dots are being studied intensively because of their potential applications to optoelectronics, quantum cryptography and quantum computing (Petroff, 2001). A number of methods for calculating the eigenstates of electrons and holes in self-assembled dots have been developed (Grundmann, 1995; Cusack, 1996; Barker, 1999; Barker, 2000) but most of the techniques used so far involve very large scale computations. As applications of dots become more important it will be necessary to go beyond the computation of energies and eigenstates and develop techniques to compute other physical properties of dots, such as relaxation times. The calculation of the eigenstates is just one step in the problem of calculating the physical properties of a dot so it is advantageous to have a method which generates them efficiently. The pur- 
pose of this work is to review one particular approach to efficient calculation of electron states (Roy, 2003) together with its application to Auger relaxation (Chaney, 2003).

One of the reasons why the calculation of self-assembled dot states involves large scale computations is that there is no natural basis in which to expand the dot eigenstates. The problem can of course be solved by expanding in any basis set, such as plane waves, but this generally leads to Hamiltonian matrices of very large dimension and makes the computation expensive. We have developed an alternative approach in which a harmonic oscillator basis is used and its parameters are optimised variationally before the Hamiltonian matrix is found. This generally leads to a large reduction in the number of basis states needed to represent the dot states accurately because the low lying basis states (i.e. the ones with a few nodes) are localised on a length scale similar to the real dot states. In section 1.2 we describe our approach and detail the dot models to which it has been applied.

Following the discussion of dot states we consider Auger relaxation rates in section 1.3. Until now, most work on Auger relaxation has involved model dot states with rates calculated in the dipole approximation (Uskov, 1997). At first sight the dipole approximation should be very accurate because of the small spatial extent of the dot states but we have found a surprising discrepancy between exact Auger rates and rates calculated in the dipole approximation. The nature of this discrepancy and the reason why it occurs are detailed in section 1.3.0. Following this we give Auger rates for a number of pyramidal dot models, compare them with rates for a harmonic oscillator model and comment on the suitability of this model. Our conclusions are summarised in section 1.4.

\section{Harmonic Oscillator Basis Calculation of Electron States}

\section{Dot Model and Schroedinger Equation}

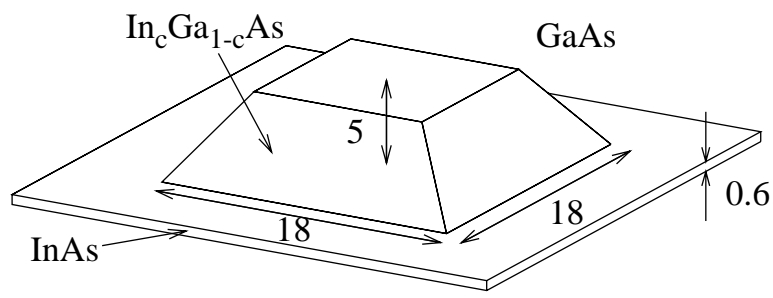

Figure 1. Dot model based on results of Bruls et al. 
The structure and composition of self-assembled dots have been under investigation for some time and knowledge of these systems is still evolving, see (Grundmann, 1995; Joyce, 1998; Liu, 2000; Fry, 2000) for example. In our work we have used scanning tunnelling microscopy results of Bruls et al (Bruls, 2002) as a starting point for constructing dot models. In this section we discuss the calculation of electron states for a particular model that we take to be typical, although not definitive, and in section 1.3 we consider Auger relaxation rates for a number of variations of this model. However our calculation method is completely general and can be used to find the quantum states of dots of arbitrary size, shape and composition.

The particular dot studied by Bruls et al (Fig. 1) is a truncated pyramid composed of $\mathrm{In}_{\mathrm{c}} \mathrm{Ga}_{1-\mathrm{c}}$ As with the Indium fraction $c$ varying linearly from 0.6 at the base to 1.0 at the top. We have no information about the composition variation in the lateral direction so we have assumed that the only composition variation is in the vertical direction. The dot base dimensions are $18 \mathrm{~nm} \times 18$ $\mathrm{nm}$, the dot height is $5 \mathrm{~nm}$ and the top face dimensions are $10.6 \mathrm{~nm} \times 10.6$ $\mathrm{nm}$. The dot stands on a $0.6 \mathrm{~nm}$ InAs wetting layer on a GaAs substrate and is surrounded by GaAs.

The dot is strained and the strain dependent effective mass and confinement potential are needed to find the quantum states. We use a commercial finite element package, Abaqus, to find the strain within the continuum elasticity approximation. The strain is calculated for a dot in the centre of a large block of material, of dimension $140 \mathrm{~nm} \times 140 \mathrm{~nm} \times 65.6 \mathrm{~nm}$, with periodic boundary conditions. The large size of the periodic cell ensures that the strain in the dot is unaffected by boundary effects. The lattice mismatch strain is taken to be $6.7 \%$, the Young's moduli and Poisson ratios for GaAs are $85.3 \mathrm{Gpa}$ and 0.32 while for InAs they are $51.4 \mathrm{Gpa}$ and 0.35 (Bruni, 1992). Values for $\mathrm{In}_{\mathrm{c}} \mathrm{Ga}_{1-\mathrm{c}} \mathrm{As}$ are obtained by linear interpolation.

The confinement potential and effective mass are found in the standard way from the strain (Fonseca, 1998). First, the electron and hole confinement potentials are found from the composition dependent conduction band offset, band gap and deformation potentials:

$$
\begin{aligned}
V_{e}(\mathbf{r}) & =V_{o}(\mathbf{r})+V_{c}(\mathbf{r}), \\
V_{o}(\mathbf{r}) & =-1.178 c(\mathbf{r})+0.381 c(\mathbf{r})^{2}, \\
V_{c}(\mathbf{r}) & =a_{c} \epsilon_{h}(\mathbf{r}), \\
V_{h h}(\mathbf{r}) & =a_{v} \epsilon_{h}(\mathbf{r})+\frac{b}{2} \epsilon_{b}(\mathbf{r}), \\
V_{l h}(\mathbf{r}) & =a_{v} \epsilon_{h}(\mathbf{r})-\frac{b}{2} \epsilon_{b}(\mathbf{r}) .
\end{aligned}
$$

Here the electron potential has been written as the sum of the conduction band offset, $V_{o}$, and a strain-dependent part, $V_{c}$, and an empirical relation (Barker, 
1999) is used to obtain the composition dependence of the conduction band offset. The energy zero is at the unstrained GaAs conduction band edge. $V_{h h}$ and $V_{l h}$ respectively are the strain dependent contributions to the heavy and light hole potentials, $\epsilon_{h}$ and $\epsilon_{b}$ respectively are hydrostatic and biaxial strains and $a_{c}, a_{v}$ and $b$ are deformation potentials and their values in $\mathrm{eV}$ are (Fonseca, 1998)

$$
\begin{aligned}
a_{c}(\mathbf{r}) & =-5.08 c(\mathbf{r})-7.17(1-c(\mathbf{r})) \\
a_{v}(\mathbf{r}) & =1.00 c(\mathbf{r})+1.16(1-c(\mathbf{r})), \\
b(\mathbf{r}) & =-1.80 c(\mathbf{r})-1.70(1-c(\mathbf{r})) .
\end{aligned}
$$

The piezoelectric contribution to the confinement potential is not included in the present work but this does not affect our conclusions about the efficiency of our method. The effect of the piezoelectric potential on the energies of the dot states is thought to be small (Grundmann, 1995).

Once the potentials are known the position dependent electron effective mass is found from first order perturbation theory (Fonseca, 1998)

$$
\begin{aligned}
m_{z}^{*}(\mathbf{r}) & =m^{*} \frac{V_{c}+E_{g}^{G a A s}-V_{l h}}{E_{g}} \\
m_{x y}^{*}(\mathbf{r}) & =m^{*} \frac{\left(V_{c}+E_{g}^{G a A s}-V_{h h}\right)\left(V_{c}+E_{g}^{G a A s}-V_{l h}\right)}{E_{g}\left(V_{c}+E_{g}^{G a A s}-\frac{3}{4} V_{l h}-\frac{1}{4} V_{h h}\right)},
\end{aligned}
$$

where $m_{z}^{*}$ and $m_{x y}^{*}$ are respectively perpendicular and lateral effective masses and $E_{g}^{G a A s}$ is the bulk GaAs band gap. $E_{g}$ and $m^{*}$ are the position dependent band gap and effective mass given by (Fonseca, 1998)

$$
\begin{aligned}
E_{g}(\mathbf{r}) & =0.41 c(\mathbf{r})+1.52(1-c(\mathbf{r})), \\
m^{*}(\mathbf{r}) & =0.023 c(\mathbf{r})+0.067(1-c(\mathbf{r})) .
\end{aligned}
$$

Within the single band effective mass approximation the Schroedinger equation for the electron states is then

$$
H \Psi=\left[\frac{1}{2}(-i \hbar \nabla+e \mathbf{A}) M^{-1}(-i \hbar \nabla+e \mathbf{A})+V(\mathbf{r})\right] \Psi=E \Psi
$$

where $M$ is the effective mass tensor and we have allowed for the possibility that the dot is in a magnetic field.

\section{Harmonic Oscillator Basis for Solution of Schroedinger Equation}

The Schroedinger equation can always be solved by expanding the wave function in a suitable orthonormal basis, that is by putting

$$
\Psi(\mathbf{r})=\sum_{i} a_{i} \psi_{i}(\mathbf{r})
$$


where the $\psi_{i}$ are the basis states. Usually it is advantageous to choose the basis to be the eigenstates of a Hamiltonian that is close to the one of interest so that the equation $H \Psi=E \Psi$ becomes $\left(H_{0}+\Delta H\right) \Psi=E \Psi$ and the basis states are chosen to be the eigenstates of $H_{0}$. If $\Delta H$ is in some sense small the eigenstates of $H$ can be found with only a few terms in the basis set expansion and the process is computationally efficient. In many cases there is a natural separation of $H$ into $H_{0}+\Delta H$, where the eigenstates of $H_{0}$ are easy to find and $\Delta H$ is small, but this does not happen for self-assembled dots because of the complicated nature of the confinement potential. Instead most authors have used a plane wave basis (Cusack, 1996; Barker, 1999; Barker, 2000) or a real space mesh (Grundmann, 1995; Stier, 1999) to find the dot states, however both approaches are computationally expensive.

In our alternative approach the basis is optimised before use so that the lowest few basis states (the ones with only a few nodes) are localised on about the same length scale as the dot states. We take the basis states to be products of lateral and vertical harmonic oscillator states so that

$$
\begin{aligned}
& \psi_{i}(\mathbf{r})=\Phi_{l_{i}}(\phi) Z_{m_{i}}(z) R_{n_{i} l_{i}}(r), \\
& \Phi_{l_{i}}(\phi)=\frac{1}{\sqrt{2 \pi}} e^{i l_{i} \phi}, \\
& Z_{m_{i}}(z)=\left(\frac{1}{2^{m_{i}} m_{i} ! \lambda_{z} \sqrt{\pi}}\right)^{\frac{1}{2}} H_{m_{i}}\left[\frac{\left(z-z_{o}\right)}{\lambda_{z}}\right] e^{-\left(z-z_{o}\right)^{2} / 2 \lambda_{z}^{2}}, \\
& R_{n_{i} l_{i}}(r)=\left(\frac{n_{i} !}{\lambda_{r}^{2}\left(n_{i}+\left|l_{i}\right|\right) !}\right)^{\frac{1}{2}} e^{-r^{2} / 4 \lambda_{r}^{2}}\left(\frac{r}{\sqrt{2} \lambda_{r}}\right)^{\left|l_{i}\right|} L_{n_{i}}^{\left|l_{i}\right|}\left[\frac{r^{2}}{2 \lambda_{r}^{2}}\right] \text {. }
\end{aligned}
$$

Here $H_{m_{i}}$ is a Hermite polynomial, $L_{n_{i}}^{\left|l_{i}\right|}$ is a Laguerre polynomial, $\lambda_{z}$ and $\lambda_{r}$ are vertical and lateral length parameters and $z_{o}$ is an offset parameter. These basis states form a complete orthonormal set for all values of $\lambda_{z}, \lambda_{r}$ and $z_{o}$ so the sum in Eq. (6) always converges to an exact dot state provided enough terms are included. We reduce the number of terms by choosing the length and offset parameters to minimise $\left\langle\psi_{0}|H| \psi_{0}>\right.$, where $\psi_{0}$ has quantum numbers $l=0$, $m=0$ and $n=0$. Thus $\psi_{0}$ is a variational approximation to the exact dot ground state and the length scale of the oscillator states is approximately the same as that of the exact dot states. This enables us to obtain accurate dot states by retaining relatively few oscillator states in the basis state expansion. The dot states and energies are found in the usual way by numerically diagonalising the resulting Hamiltonian matrix. A second advantage of the harmonic oscillator basis is that the effect of a perpendicular magnetic field can be included with relatively little additional computational effort. This is explained in our earlier paper (Roy, 2003) which also gives full details of our method. 


\section{Comparison of Harmonic Oscillator and Plane Wave Methods}

Table 1. Comparison of energies calculated with the harmonic oscillator and plane wave bases.

\begin{tabular}{clll}
\hline State & Energy $(\mathrm{eV})$ & Energy $(\mathrm{eV})$ & $\%$ difference \\
\hline 1 & -0.2247 & -0.2234 & 0.6 \\
2 & -0.1563 & -0.1564 & 0.1 \\
3 & -0.1563 & -0.1564 & 0.1 \\
4 & -0.0947 & -0.0960 & 1.4 \\
5 & -0.0720 & -0.0726 & 0.8 \\
6 & -0.0594 & -0.0606 & 2.0 \\
\hline
\end{tabular}

Energies of electron states for the dot shown in Fig. 1, calculated with the harmonic oscillator and plane wave expansions, are given in table 1. The harmonic oscillator results were obtained with $l_{\max }=12, m_{\max }=20$ and $n_{\max }=20$ which corresponds to a total of 11,025 basis states. The plane wave results are for for the same dot in a $60 \mathrm{~nm} \times 60 \mathrm{~nm} \times 60 \mathrm{~nm}$ periodic cell. 41 plane waves are used in the vertical direction and 21 in each of the two lateral directions which corresponds to a total of 18,081 basis states. The ground state energy calculated with the harmonic oscillator basis is accurate to about $0.1 \mathrm{meV}$ but the plane wave ground state energy is about $0.6 \%$ higher. The agreement between the two sets of results very good, with only a small discrepancy that results from a combination of the poorer convergence of the plane wave method and the difficulty of calculating the plane wave Hamiltonian matrix elements accurately (Roy, 2003).

The superior convergence of the harmonic oscillator basis calculation is illustrated in Fig. 2. The figure shows the convergence of the energies of the states that are bound in the dot. It is clear that the convergence of the harmonic oscillator basis calculation is the fastest. The accuracy needed for meaningful comparison with experiment is about $1 \mathrm{meV}$ or better. This can be achieved with the harmonic oscillator basis with just 324 basis states $\left(l_{\max }=\right.$ 4, $m_{\max }=8$ and $n_{\max }=3$ ) while the plane wave calculation requires 7,425 basis states (33 in the vertical direction and 15 in each of the lateral directions) for the same accuracy. Since the CPU time needed to diagonalise a $N \times N$ matrix scales like $N^{3}$ this leads to an enormous reduction in CPU time.

Another difficulty with the plane wave method is that the results are sensitive to the size of the periodic cell used for the calculation. Care has to be taken to ensure that the tail of the wave function is sufficiently small at the cell boundary and ground state energies can be around $10 \%$ too low if this condition is not satisfied. See (Roy, 2003) for details. 


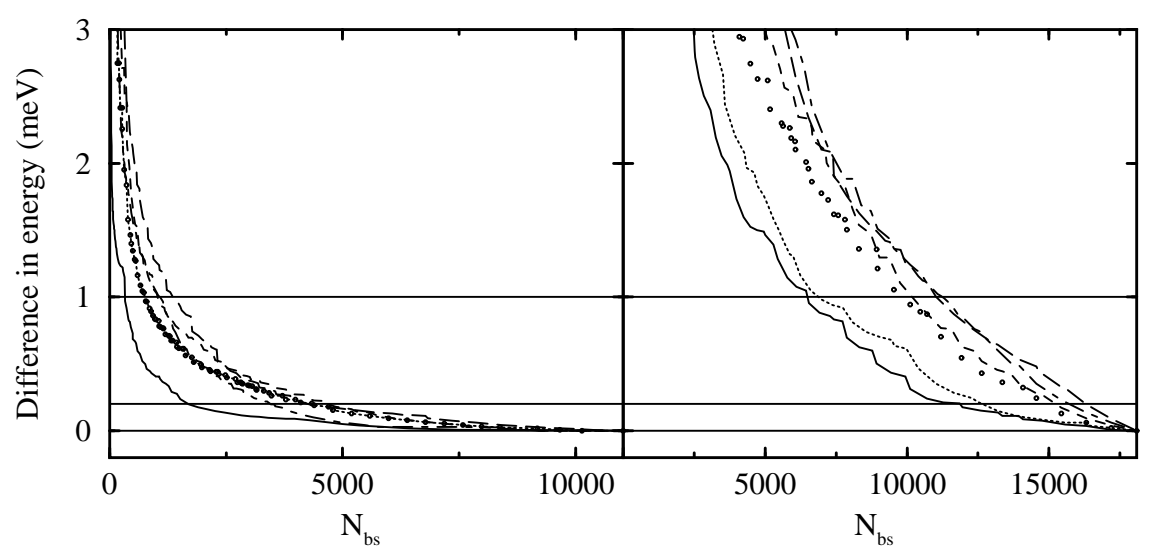

Figure 2. Rate of convergence of bound states as a function of number of basis functions included in the calculation. Solid line (ground state), dotted line (state 2), points (state 3), dashed line (state 4), long dashed line (state 5), dot dash line (state 6). Horizontal solid lines show energy differences of $0,0.2 \mathrm{meV}$ and $1 \mathrm{meV}$ between the energy calculated with the largest number of basis functions and the energies calculated with $N_{b s}$ basis functions. Left frame: harmonic oscillator basis; right frame: plane wave basis.

\section{Auger Relaxation}

\section{Auger Processes and the Dipole Approximation}

The rate of relaxation of a dot electron in an excited state down to the ground state is a quantity that is very relevant to the operation of optoelectronic devices (Morris, 1999; Petroff, 2001). When the electron or hole density outside the dot, either in the capping material or in the wetting layer, is sufficiently high the dominant relaxation process is expected to be an Auger process where energy is transfered from the electron in the dot to the external particle. We consider Auger processes that involve electrons external to the dot, both in the wetting layer and the capping layer.

The rate of Auger transitions is found from the Fermi Golden Rule in the standard way by summing over all the transitions that conserve energy:

$$
\frac{2 \pi}{\hbar} \sum_{\mathbf{k}_{i}, \mathbf{k}_{f}}\left|\left\langle\Psi_{f}|W| \Psi_{i}\right\rangle\right|^{2} f\left(k_{i}\right) \delta\left(E\left(\mathbf{k}_{f}\right)-E\left(\mathbf{k}_{i}\right)+\Delta E_{Q D}\right),
$$

where $\Psi_{i}$ and $\Psi_{f}$ are the initial and final states. Exchange effects are neglected which allows us to take these states to be products of dot states and external electron states. The external electron states are taken to be either $2 \mathrm{D}$ or $3 \mathrm{D}$ plane waves. In either case $\mathbf{k}_{i}$ and $\mathbf{k}_{f}$ are the initial and final wave-vectors of the external electrons and $\Delta E_{Q D}$ is the change in energy of the dot electron. $f\left(k_{i}\right)$ is the energy distribution function of the external electrons in their initial 
state and we take it to be a Maxwell-Boltzmann distribution. We suppose that the dot electron interacts with the external electron via the screened Coulomb interaction,

$$
W\left(\mathbf{r}-\mathbf{r}_{e}\right)=\frac{e^{2}}{4 \pi \epsilon \epsilon_{0}\left|\mathbf{r}-\mathbf{r}_{e}\right|} \exp \left(-\kappa\left|\mathbf{r}-\mathbf{r}_{e}\right|\right)
$$

where $\mathbf{r}$ and $\mathbf{r}_{e}$ are the dot and external electron coordinates, $\epsilon$ is the dielectric constant, $\kappa=\sqrt{4 \pi e^{2} n_{B} /\left(\epsilon \epsilon_{0} k_{B} T_{B}\right)}$ is the Debye screening constant, and $n_{B}$ and $T_{B}$ are the bulk electron density and temperature. The screening effect of the wetting layer electrons is neglected (Uskov, 1997).

To calculate the relaxation rate from Eq. (8) we need to know the electron densities in the wetting layer and the bulk. We suppose that electrons are injected into the bulk conduction band and relax down to the conduction band edge. After this they can be captured into the wetting layer and then recombine with holes or they can recombine directly from the conduction band. This process is described by the following rate equations

$$
\begin{aligned}
& \frac{d n_{p}(t)}{d t}=G(t)-\frac{n_{p}(t)}{\tau_{r e l}} \\
& \frac{d n_{1}(t)}{d t}=\frac{n_{p}(t)}{\tau_{r e l}}-\frac{n_{1}(t)}{\tau_{r 1}}-\frac{n_{1}(t)}{\tau_{c a p}} \\
& \frac{d n_{W L}(t)}{d t}=\frac{n_{1}(t)}{\tau_{c a p}}-\frac{n_{W L}(t)}{\tau_{r 2}}
\end{aligned}
$$

where the time constants are taken from experimental data of Liu et al (Liu, 2001) $\left(\tau_{\text {rel }}=2 \mathrm{ps}, \tau_{\text {cap }}=7 \mathrm{ps}, \tau_{r 1}=1 \mathrm{~ns}, \tau_{r 2}=0.25 \mathrm{~ns}\right)$ and the injection rate $G(t)$ is chosen to give the desired number of injected electrons.

Auger relaxation rate calculations for electrons in a self-assembled dot were first performed by Uskov et al (Uskov, 1997). They used an axially symmetric dot model so the dot states contain an angular momentum factor, identical to the factor $\Phi_{l_{i}}$ that appears in the basis states defined in Eq. (7). In addition they approximated the interaction potential by Taylor expanding it about $\mathbf{r}=\mathbf{0}$ so

$$
W\left(\mathbf{r}-\mathbf{r}_{e}\right) \sim W\left(-\mathbf{r}_{\mathbf{e}}\right)+\mathbf{r} \cdot \nabla W\left(-\mathbf{r}_{\mathbf{e}}\right) \ldots
$$

At first sight this is physically reasonable because the spatial extent of the external electron state is much greater than that of the localised dot electron state, so that typically $r<<r_{e}$. The first term in the Taylor expansion does not contribute to the relaxation rate because of the orthogonality of the initial and final states. So if only the second term is retained the matrix element for the transition becomes a dipole matrix element and the approximation is equivalent to the dipole approximation used in atomic physics to find radiative transition rates. The advantage of making this approximation is that the Auger 
relaxation rate can be found analytically in some cases but we have found a discrepancy between the exact and dipole relaxation rates.

\section{Breakdown of the Dipole Approximation}

We have examined the validity of making the dipole approximation for the lateral coordinates, in other words making the Taylor expansion with respect to $x$ and $y$ only. We use an axially symmetric dot model and take the dot states to be products of lateral and vertical harmonic oscillator states. That is, the states we use for testing the dipole approximation are identical to the basis states defined in Eq. (7). Instead of Taylor expanding the interaction potential in real space we work with its Fourier representation,

$$
\begin{aligned}
W\left(\mathbf{r}-\mathbf{r}_{e}\right) & =\frac{2 \pi}{A} \frac{e^{2}}{4 \pi \epsilon \epsilon_{0}} \sum_{\mathbf{p}}\left(\kappa^{2}+p^{2}\right)^{-1 / 2} \\
& \times \exp \left(-\left|z-z_{e}\right| \sqrt{\kappa^{2}+p^{2}}+i \mathbf{p} \cdot\left(\mathbf{r}-\mathbf{r}_{e}\right)\right),
\end{aligned}
$$

where $\mathbf{p}$ is a two dimensional vector in the $x, y$ plane, the system is taken to be periodic with area $A$, and $z_{e}$ is the vertical position of the wetting layer. The exact matrix element for an Auger process in which the momentum change of a wetting layer electron is $\hbar \mathbf{q}=\hbar\left(\mathbf{k}_{f}-\mathbf{k}_{i}\right)$ is then

$$
\begin{aligned}
& \frac{2 \pi}{A} \frac{e^{2}}{4 \pi \epsilon \epsilon_{0}} \frac{1}{\left(\kappa^{2}+q^{2}\right)^{1 / 2}} \\
\times & \left\langle\psi_{f}\left|\exp \left(-\left|z-z_{e}\right| \sqrt{\kappa^{2}+q^{2}}\right) \exp (-i \mathbf{q} \cdot \mathbf{r})\right| \psi_{i}\right\rangle,
\end{aligned}
$$

while the dipole approximation is made by expanding the second exponential factor as $\exp (-i \mathbf{q} \cdot \mathbf{r}) \sim 1-i \mathbf{q} \cdot \mathbf{r} \ldots$. Here $\psi_{i}$ and $\psi_{f}$ are initial and final dot states of the form defined by Eq. (7).

In the case when the initial state is the first excited state of lateral motion and the final state is the ground state the exact matrix element is

$$
\frac{2 \pi}{A} \frac{e^{2}}{4 \pi \epsilon \epsilon_{0}} \frac{1}{\left(\kappa^{2}+q^{2}\right)^{1 / 2}} \times F(q) \frac{q \lambda_{r}}{i \sqrt{2}} \exp \left(-q^{2} \lambda_{r}^{2} / 2\right),
$$

where $F(q)$ is the matrix element between vertical states:

$$
F(q)=\left\langle Z_{f}\left|\exp \left(-\left|z-z_{e}\right| \sqrt{\kappa^{2}+q^{2}}\right)\right| Z_{i}\right\rangle,
$$

which do not change in the case we are considering. The corresponding matrix element in the dipole approximation is

$$
\frac{2 \pi}{A} \frac{e^{2}}{4 \pi \epsilon \epsilon_{0}} \frac{1}{\left(\kappa^{2}+q^{2}\right)^{1 / 2}} \times F(q) \frac{q \lambda_{r}}{i \sqrt{2}}\left[1-q^{2} \lambda_{r}^{2} / 2 \ldots\right] .
$$


Strictly speaking, the dipole approximation corresponds to the first term in the square brackets but we have retained terms up to cubic order in $\mathbf{q} \cdot \mathbf{r}$ to allow comparison of the exact and approximate matrix elements. It is clear that the term in square brackets in the approximate matrix element is just the power series expansion of the exponential factor in the exact matrix element. Therefore the approximation is valid when $\lambda_{r}^{2} q^{2} / 2<<1$. When the temperature is low and the energy transfer is high, $k_{f}>>k_{i}$ so $|\mathbf{q}|=\left|\mathbf{k}_{f}-\mathbf{k}_{i}\right| \sim k_{f}$ and $\Delta E_{Q D}=\hbar \omega \sim \hbar^{2} q^{2} / 2 m^{*}$. In addition, $\lambda_{r}$ and $\omega$ for the harmonic oscillator states are related via $\lambda_{r}^{2}=\hbar / 2 m^{*} \omega$ therefore $\lambda_{r} q \sim 1$. Thus the dipole approximation is never valid no matter how small the dot is. A similar result holds in the case of relaxation of electrons in harmonic oscillator states interacting with bulk electrons. It is also likely that the dipole approximation is not valid for real dot states. The lateral length scale of a typical dot state is of order $l=\sqrt{\left\langle r^{2}>-\langle r\rangle^{2}\right.}$. We have calculated the product $l q$ for a number of pyramidal dot models and found that it is typically of order 1 . This suggests that the dipole approximation is typically not valid but as there is no advantage to using the approximation when the matrix elements are calculated numerically we have not pursued this issue.

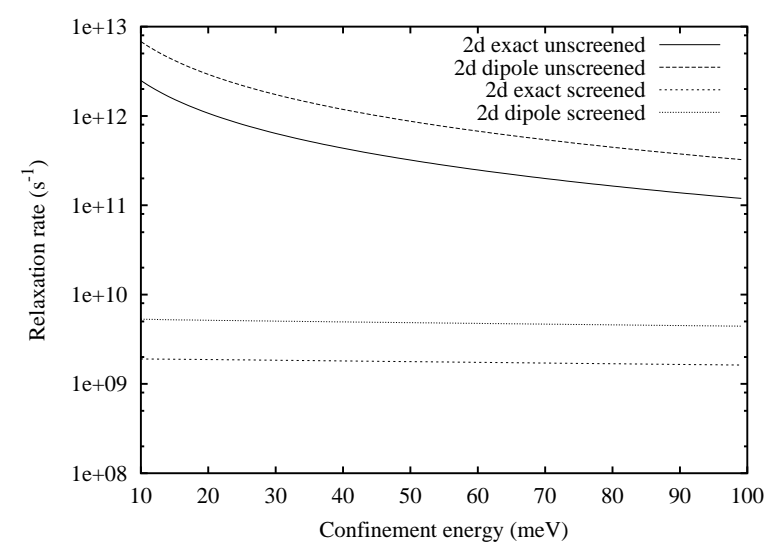

Figure 3. Relaxation rates for a dot electron in the first excited lateral state interacting with wetting layer electrons. Rates are shown for the harmonic oscillator model as a function of lateral confinement energy.

Quantitatively, the discrepancy between the exact and dipole relaxation rates is a constant factor. This is illustrated in Figs. 3 and 4 which show exact and dipole rates for relaxation of an electron in the first lateral excited state of a harmonic oscillator as a function of lateral confinement energy $\hbar \omega$. The rates were computed by numerically evaluating the sum in Eq. (8) as a multidimen- 


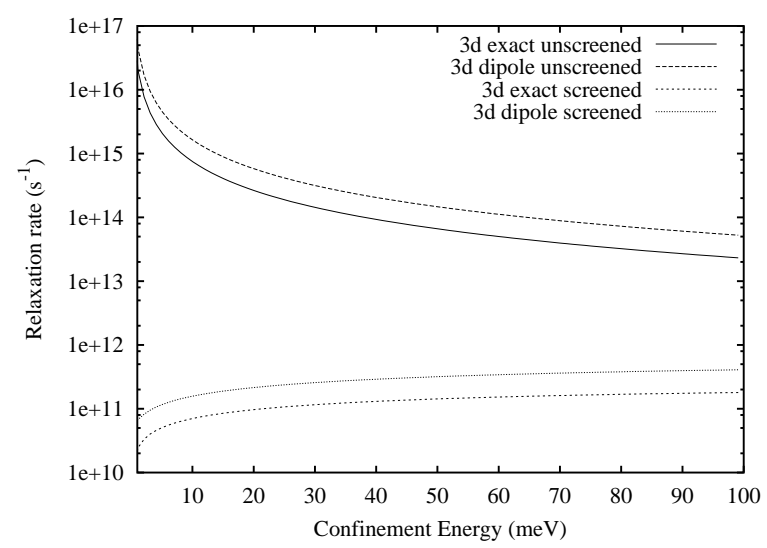

Figure 4. Relaxation rates for a dot electron in the first excited lateral state interacting with bulk electrons. Rates are shown for the harmonic oscillator model as a function of lateral confinement energy.

sional integral to an accuracy of about $0.1 \%$. The vertical length parameter is $2.41 \mathrm{~nm}$, the distance to the wetting layer is $2.5 \mathrm{~nm}$, the material is InAs $\left(m^{*}=0.023, \epsilon=15.15\right)$, the bulk electron temperature is $1 \mathrm{~K}$ and the injected electron density is $10^{22} \mathrm{~m}^{-3}$. Rates for screened and unscreened interactions are given. Qualitatively, the dipole rates show the same trends as the exact ones in all cases but quantitatively they differ from the exact rates by a factor of order $2-3$ that is nearly independent of the confinement energy. This is consistent with the arguments in the previous paragraph. The lack of dependence on confinement energy occurs because the product $\lambda_{r} q$ is approximately constant. The numerical value of the factor can be understood by considering the matrix element. The exact and dipole matrix elements differ by the factor $\exp \left(-\lambda_{r}^{2} q^{2} / 2\right) \sim \exp (-0.5)$. Therefore the rates differ by a factor of order $e$ as found numerically. The dependence of the harmonic oscillator model relaxation rates on confinement energy can be understood by considering the $q$ dependence of the matrix elements, as detailed by (Chaney, 2003).

\section{Effect of Dot Geometry on Relaxation Rate}

To examine the effect of dot geometry on the Auger relaxation from the first excited state to the ground state we have computed the relaxation rate as a function of electron density for a number of pyramidal dot models (Chaney, 2003). The models include square and rectangular based truncated pyramids and a square based full pyramid, dimensions are given in table 2 . In each case the dot is taken to be composed of InAs. The small effect of different 
Table 2. Dimensions of quantum dot models used in this work.

\begin{tabular}{lr}
\hline Model geometry & $l \times w \times h(\mathrm{~nm})$ \\
\hline Square based full pyramid & $6.0 \times 6.0 \times 6.0$ \\
Rectangular based truncated pyramid & $14.0 \times 21.0 \times 5.0$ \\
Square based truncated pyramid & $17.7 \times 17.7 \times 5.0$ \\
\hline
\end{tabular}

dielectric constants of the dot and the capping material is ignored. As harmonic oscillator states have been proposed as a model for self-assembled dot states (see (Petroff, 2001) for a review) we have also computed rates for the harmonic oscillator model. The results are shown in Figs. 5 (interaction with wetting layer electrons) and 6 (interaction with bulk electrons). The temperature is 1 $\mathrm{K}$. The injected electron density range is the same for the calculations leading to Figs. 5 and 6 so the figures are directly comparable. All of the curves have a similar structure: the rate first increases with electron density because the increasing density increases the probability of an interaction and then decreases because of screening.

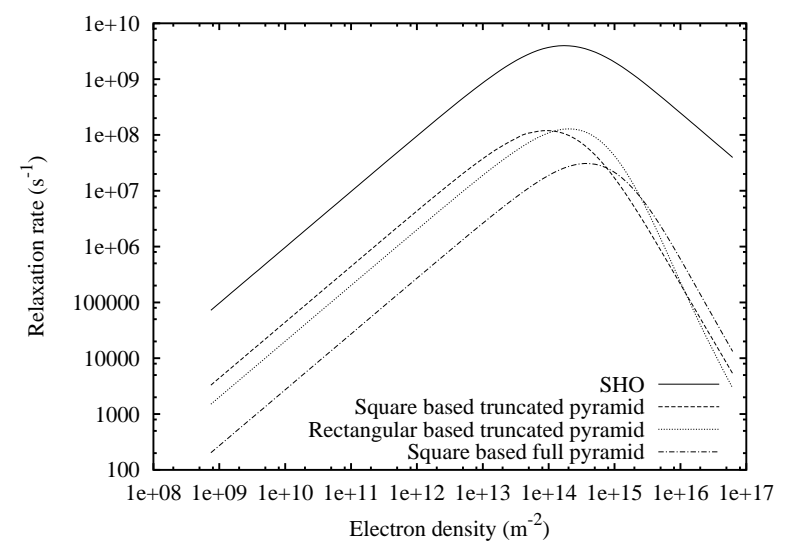

Figure 5. Relaxation rates as a function of wetting layer electron density for a dot electron in the first excited lateral state interacting with wetting layer electrons.

The length parameters for the harmonic oscillator model have been chosen to be similar to the radial and vertical RMS displacements, $\sqrt{\left\langle r^{2}\right\rangle-\langle r\rangle^{2}}$ and $\sqrt{\left\langle z^{2}\right\rangle-\langle z\rangle^{2}}$ of the pyramidal models. The vertical length parameter is $2.24 \mathrm{~nm}$, the lateral length parameter is $4.5 \mathrm{~nm}$ and all other parameters are as in the previous section. In the case of relaxation via interactions with wetting layer electrons (Fig. 5) the harmonic oscillator model rate is around 


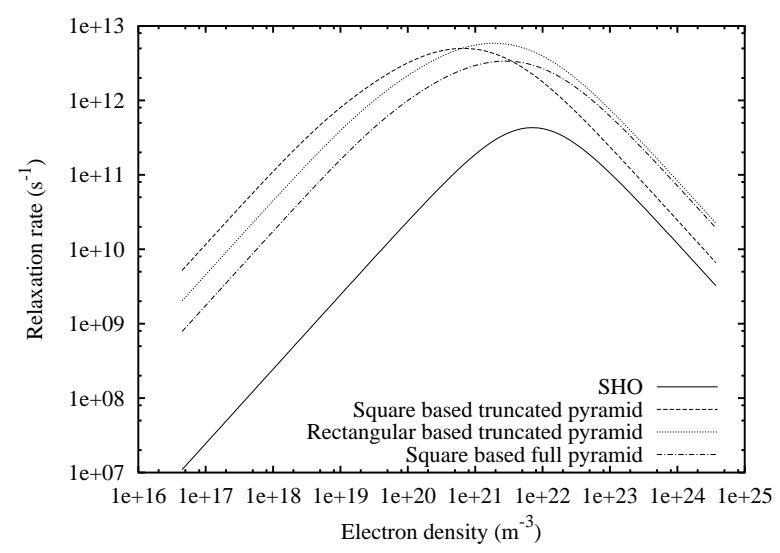

Figure 6. Relaxation rates as a function of bulk electron density for a dot electron in the first excited lateral state interacting with bulk electrons.

one to two orders of magnitude larger than any of the pyramidal model rates. This is a consequence of the fact that a harmonic oscillator potential does not confine electrons as effectively as the potential in the pyramidal model. This potential rises abruptly at the dot boundary. Consequently the pyramidal dot states have a smaller overlap than the harmonic oscillator states with the wetting layer. On the other hand, in the case of relaxation via interactions with bulk electrons (Fig. 6) the harmonic oscillator model rate is around one to two orders of magnitude smaller than any of the pyramidal model rates. This is a consequence of the form of the matrix element for interactions with bulk electrons,

$$
\frac{4 \pi}{V} \frac{e^{2}}{4 \pi \epsilon \epsilon_{0}} \frac{1}{\left(\kappa^{2}+q^{2}\right)}\left\langle\psi_{f}|\exp (-i \mathbf{q} \cdot \mathbf{r})| \psi_{i}\right\rangle
$$

where $V$ is the volume of the system and $\hbar \mathbf{q}$ is a 3D momentum change. As explained in the previous section $q^{2} \sim 2 m^{*} \Delta E / \hbar^{2}$ at low temperature so the matrix element is of order $1 / \Delta E$ in the low density, low screening limit $(\kappa \sim 0)$. $\Delta E$ for the harmonic oscillator model is about 2 to 3 times larger than for the pyramidal models and this results in the rate being reduced by about an order of magnitude. In addition, there is some further reduction through the value of the matrix element of $\exp (-i \mathbf{q} \cdot \mathbf{r})$. This result illustrates the difficulty of reproducing self-assembled dot physics accurately with a harmonic oscillator model. The length parameter and excitation gap of the harmonic oscillator are not independent so it is not possible to match the length scale and gap of the pyramidal dot states accurately with an oscillator model, however the oscillator 
model does reproduce the Auger relaxation physics qualitatively, as illustrated in Figs. 5 and 6.

Figs. 5 and 6 also show that there is considerable variability in the rates for the various pyramid models, particularly at low density. The rate for relaxation via bulk interactions is always the highest but the ratio of the bulk and wetting layer relaxation rates is very sensitive to the time constants in Eq. (10) and these time constants are probably system dependent. Composition variations are likely to decrease the relaxation rate for wetting layer interactions by a small factor because a composition gradient with the largest In fraction at the top of the dot causes a small shift of the electron wave function away from the wetting layer. Some of the relaxation rates predicted here are comparable to those measured experimentally by Morris et al (Morris, 1999) however it is difficult to make a quantitative comparison with these results because the electron densities corresponding to the experimental conditions of Morris et al are not known. It would be very interesting to have experimental data for a system in which both the relaxation rate and the electron densities have been measured.

\section{Conclusion}

We have reviewed an efficient method for computation of quantum states in self-assembled dots and its application to Auger relaxation. Our method enables dot states to be computed economically within the effective mass approximation and seems to be useful for computing physical properties of dots. Our results on Auger relaxation indicate that the dipole approximation does not give an accurate description of the Auger relaxation rate. We have also indicated the difficulties of using harmonic oscillator states as model states for calculating Auger rates. The difficulties of using the harmonic oscillator model to describe self-assembled dots containing more than a few electrons have also been discussed by Petroff et al (Petroff, 2001) and in both cases the difficulties are caused by the very simple excitation spectrum of the harmonic oscillator model. Our theory predicts relaxation rates can be around $10 \mathrm{ps}$, as seen experimentally, but the rates depend critically on the bulk and wetting layer electron densities and it would be very useful to have measurements of Auger relaxation rates in a system for which these electron densities are known.

\section{Acknowledgements}

The numerical calculations described here were performed on a supercomputer at the Leicester Mathematical Modelling Centre which was purchased through the EPSRC strategic equipment initiative. DC is grateful for the award of an EPSRC research studentship. 


\section{References}

Barker, J. A. and E. P. O'Reilly (1999). "The influence of inter-diffusion on electron states in quantum dots," Physica E 4, 231.

Barker, J. A. and E. P. O'Reilly (2000). "Theoretical analysis of electron-hole alignment in InAs-GaAs quantum dots," Phys. Rev. B. 61, 13840.

Bruls, D. M., J. W. A. M. Vugs, P. M. Koenraad, M. S. Skolnick, M. Hopkinson, F. Long, S. P. A. Gill and J. H. Wolter (2002). "Determination of the shape and indium distribution of lowgrowth-rate InAs quantum dots by cross-sectional scanning tunnelling microscopy," Appl. Phys. Lett. 81, 1708.

Bruni, M. R., A. Lapiccirella, G. Scavia, M. G. Simeone, S. Viticoli, and N. Tomassini (1992). "Thermodynamic study of molecular-beam epitaxial-growth of InGaAs/GaAs strained layer superlattices," Thermochemica Acta., 210, 49.

Chaney, D., M. Roy, P. A. Maksym and F. Long (2003). "The effect of self-assembled quantum dot geometry on Auger relaxation rate," Proc. 26th Int. Conf. on the Physics of Semiconductors, Eds. A. R. Long and J. H. Davies, Bristol, IOP Publishing.

Cusack, M. A., P. R. Briddon and M. Jaros (1996). "Electronic structure of InAs/GaAs selfassembled quantum dots," Phys. Rev. B. 54, R2300.

Fry P.W., I. E. Itskevich , D. J. Mowbray, M. S. Skolnick, J. J. Finley, J. A. Barker, E. P. O'Reilly, L. R. Wilson, I. A. Larkin, P. A. Maksym, M. Hopkinson, M. Al-Khafaji, J. P. R. David, A. G. Cullis, G. Hill and J. C. Clark (2000). "Inverted electron-hole alignment in InAs-GaAs self-assembled quantum dots," Phys. Rev. Lett. 84733.

Fonseca, L. R. C., J. L. Jimenez, J. P. Leburton and R. M. Martin 1998). "Self-consistent calculation of the electronic structure and electron-electron interaction in self-assembled InAsGaAs quantum dot structures," Phys. Rev. B 57, 4017.

Grundmann, M., O. Stier and D. Bimberg (1995). "InAs/GaAs pyramidal quantum dots - strain distribution, optical phonons, and electronic-structure," Phys. Rev. B 52, 11969.

Joyce P.B., T.J. Krzyzewski, G. R. Bell, B. A. Joyce and T. S. Jones (1998). "Composition of InAs quantum dots on GaAs(001): Direct evidence for (In,Ga)As alloying," Phys. Rev. B 58 R15981.

Liu, B., Q. li, Z. Xu and W. E. Ge (2001). "Detection of efficient carrier capture in ultrathin InAs/GaAs layers using a degenerate pump-probe technique," J. Phys: Condens. Matter 13 3923.

Liu, N., J. Tersoff, O. Baklenov, A. L. Holmes and C. K. Shih (2000). "Nonuniform composition profile in $\mathrm{In}_{0.5} \mathrm{Ga}_{0.5}$ As alloy quantum dots," Phys. Rev. Lett. 84, 334.

Morris, D., N. Perret and S. Fafard (1999). "Carrier energy relaxation by means of Auger processes in InAs/InGaAs self-assembled quantum dots," Appl. Phys. Lett. 75, 3593.

Petroff, P. M., A. Lorke and A. Imamoglu (2001). "Epitaxially self-assembled quantum dots," Physics Today 54 Is. 5, 46.

Roy M., and P. A. Maksym (2003). "An efficient method for calculating electronic states in self-assembled quantum dots," submitted to Phys. Rev. B.

Stier, O., M. Grundmann and D. Bimberg (1999). "Electronic and optical properties of strained quantum dots modelled by 8 -band $\mathbf{k} \cdot \mathbf{p}$ theory," Phys. Rev. B 59, 5688.

Uskov, A. V., F. Adler, H. Schweizer and M. H. Pikuhn (1997). "Auger carrier relaxation in self-assembled quantum dots by collisions with two-dimensional carriers," J. Appl. Phys., 81, 7895 